\title{
Diagnostic Techniques for Soil-Transmitted Helminths - Recent Advances
}

\author{
Sumeeta Khurana $\mathbb{D}^{\prime}$ \\ Shreya Singh ${ }^{2}$ \\ Abhishek Mewara' \\ 'Department of Medical Parasitology, \\ Post Graduate Institute of Medical \\ Education and Research, Chandigarh, \\ India; ${ }^{2}$ Department of Medical \\ Microbiology, Post Graduate Institute of \\ Medical Education and Research, \\ Chandigarh, India
}

\begin{abstract}
Soil-transmitted helminth (STH) infections (hookworms, Trichuris, Ascaris) and Strongyloides spp. are associated with a substantial global burden and high morbidity. Sensitive and specific methods for diagnosis of these infections are essential for mapping the burden in communities, accurate assessment of infection levels, to guide interventions and monitoring the success of STH control programs. Despite considerable progress to control STH over several decades, we are still far from identifying a fully adequate diagnostic test. Conventional microscopy-based methods such as direct Kato-Katz smear or mounts after stool centrifugation/ flotation-based concentration techniques have been the mainstay of diagnosis, especially in resource-poor countries where these infections abound. However, recently, these are being adapted to closed, easy to perform, digital formats, thereby improving the sensitivity as well as applicability in a remote, resource-limited setting. The use of image analysis systems to identify and quantify helminth eggs, with potential adaptation to smartphones, is also promising. Antibody detection tests have a limited role mostly in the case of Strongyloides hyperinfection. Coproantigen detection tests have been developed and used in veterinary practice for detection of STH, but these have not been evaluated for use in humans. More sensitive molecular diagnostics, including assays developed with new bioinformatic tools and techniques such as polymerase chain reaction (PCR), quantitative PCR (qPCR) and loop-mediated amplification assay, can help in the clear and precise assessment of STH burden during elimination phase and are of immense value for diagnosis in areas with low endemicity and in travelers to endemic regions. Moreover, the molecular techniques will help detect new species that may emerge. Sample preservation and efficient DNA extraction are critical and significantly affect the efficiency of molecular diagnostic tests. In addition to the diagnosis of clinical or asymptomatic infection in humans, detection of STH eggs in environmental samples is imperative to boost STH control efforts. Overall the diagnostic performance, cost-effectiveness, ease of performance, rapidity and in-field applicability of any test should be considered when choosing from the various diagnostic assays in areas with different endemicity, in addition to striving towards the development of novel technologies and optimization of existing methods.
\end{abstract}

Keywords: soil-transmitted helminths, diagnosis, microscopy, molecular

\section{Introduction}

More than 1.5 billion people, comprising nearly a quarter of the global population, are infected with soil-transmitted helminths (STH). ${ }^{1}$ The STH infections are widely distributed in tropical and subtropical areas of the world, with the highest prevalence reported from Sub-Saharan Africa, China, South America and Asia. More than 267 million children of preschool-age and 568 million school-age children reside in these areas and need therapeutic and preventive interventions. ${ }^{1}$ These infections are most commonly reported in people residing in or travelling to regions
Correspondence: Sumeeta Khurana Department of Medical Parasitology, Research Block A, PGIMER, Chandigarh, India

Tel +9I-98728|48I7

Fax +0 I72-274440 I

Email sumeetakhurana@hotmail.com 
with poor access to clean drinking water, sanitation and hygiene, usually in low or middle-income countries; or in vulnerable populations in high-income countries. ${ }^{2}$ The World Health Organization (WHO) has recognized infections caused by four species of STHs, viz. Ascaris lumbricoides, hookworms (i.e., Necator americanus and Ancylostoma duodenale) and Trichuris trichiura among neglected tropical diseases (NTDs). ${ }^{1}$ More recently, some areas in South East Asia have been reporting zoonotic hookworm Ancylostoma ceylanicum in addition to human hookworms. ${ }^{2}$ Although Strongyloides stercoralis is not yet considered in this list of NTD by the WHO, it is also an important STH given the significant disease morbidity caused by it and its geographic overlap with the other STHs. More than 600 million people are estimated to be infected by $S$. stercoralis globally. ${ }^{1}$

STH infections are usually clinically asymptomatic, though non-specific gastrointestinal symptoms and/or eosinophilia may be seen. Since STH seldom cause mortality, their impact on health is measured using disabilityadjusted life years (DALYs). The STH infections account for a high global burden with substantial morbidity. ${ }^{3,4}$ Recent data have shown that STH caused 2090 deaths in 2019 with 1.97 million (1.26-3.00) DALYs, in which hookworm disease was the predominant cause. ${ }^{5}$ In fact, the morbidity is directly proportional to the intensity of infection and debilitating outcomes such as anemia, intestinal obstruction, retarded physical or cognitive development are seen in heavily infected individuals, especially children. ${ }^{6-8}$ Iron deficiency anemia caused by hookworm infection during pregnancy has been linked to adverse maternal-fetal outcomes, including premature and low birth weight babies, impaired lactation, etc. ${ }^{9}$

At the turn of the new millennium, preventive chemotherapy (PC) in school-age children was endorsed by the WHO for STH control and stipulated further in the 2012 London Declaration on NTDs, with the ultimate aim to break STH transmission. ${ }^{10-12}$ The WHO recommends an integrated approach, which includes access to clean water, promote sanitation, hygiene and health education, along with mass drug administration to children, and with sustained efforts, STH elimination is expected to be achieved by $2030 .{ }^{11}$ It should be noted that each STH species has distinct epidemiological features and agedependent patterns of infection prevalence (maximum prevalence of $A$. lumbricoides and T. trichiura is usually seen in young children, whereas the peak prevalence of hookworms is observed in adolescence or early adulthood), reinfection rates (short-lived helminths reinfect more rapidly), ${ }^{12}$ and varying sensitivity to albendazole, ${ }^{13}$ which could influence the efficacy of PC strategies. ${ }^{13}$ Moreover, adults are not targeted for PC and remain important reservoirs for reinfection of treated children. Although PC is implemented without the need to assess individual infection status, accurate prevalence estimates using high sensitivity diagnostics are important to ascertain the intensity of infection in different regions. Diagnostics also inform on the performance of control measures in terms of reduction in the prevalence and/or intensity of infections, which can guide the frequency of preventive chemotherapy.

Epidemiological evidence reveals an increased prevalence of STH infections associated with direct exposure to wastewater, sludge amended soil and/or eating vegetables irrigated with them. ${ }^{14}$ Thus, apart from the detection of clinical infections, the detection of STH in such samples is also important. In this review, we elaborate on the various tools for diagnosis of STH in clinical specimens, sewage and wastewater.

Conventionally, the STH diagnosis is carried out by the examination of stool or other gastrointestinal samples for STH eggs, larvae or adult worms by microscopy. However, choice of the diagnostic technique also depends upon whether it is being employed in a high or low endemic setting since highly sensitive techniques would be required for surveillance in low endemic areas, travelers from these regions or during the elimination phase.

\section{Microscopy Based Methods}

The detection of STH by microscopy, despite being simple and low cost, lacks reasonable sensitivity due to many factors which include intermittent excretion of parasite ova, low infection intensity below the limit of detection, improper transportation or storage of sample leading to parasite disintegration etc. The parasite morphology may become distorted due to storage or drug treatment. Thus, at least three fresh fecal specimens should be examined over 10 days and some parasite concentration techniques should be employed. ${ }^{15-17}$ Various microscopy-based diagnostic tests for STH diagnosis are summarized in Figure 1.

\section{Wet Mount Preparation}

A smooth, thin preparation of stool with saline and iodine is examined for larvae or eggs of helminths. The characteristics of various STH eggs or larvae are summarized in Figure 2. 


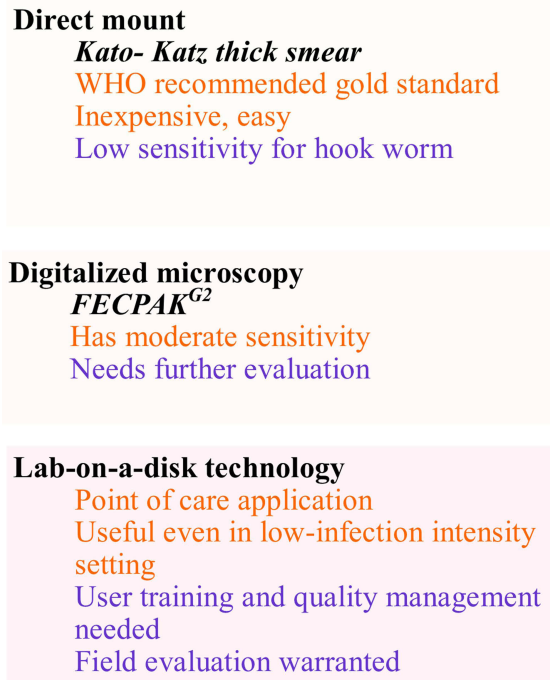

\section{Stool concentration}

Formol ether concentration (FEC)

Improves sensitivity

Can be examined in several hours

Use of ether-hazardous

Need for centrifugation

\section{Parasep ${ }^{\circledR} S F$}

Faster processing

Clearer background with less fecal debris

Better yield of T. trichura, A. lumbricoides

More expensive than FEC

\section{McMaster technique}

Inexpensive, easily implemented

Needs special counting chamber

Poor performance for unfertilized A scaris eggs

\section{FLOTAC}

High sensitivity, closed system

Mini-FLOTAC version lacks centrifugation

step

Lower average egg count estimation

Figure I Summary of the advantages (orange) and disadvantages (purple) of microscopy-based diagnostic tests for soil-transmitted helminths.

\section{Kato-Katz Thick Smear ${ }^{16}$}

Currently, the Kato-Katz (KK) method is a WHOrecommended "Gold standard" method for detection of STH eggs, and additionally for Schistosoma eggs especially when quantitation of eggs is desired. Several modifications of Kato-Katz are in use. Briefly, large particles are removed by pressing the feces through a mesh screen followed by transfer of a known amount of the sample on a slide through a template with a hole which holds a fixed amount of fecal material (e.g., a hole of $9 \mathrm{~mm}$ on a $1 \mathrm{~mm}$ thick template holds about $50 \mathrm{mg}$ of feces). The template is removed and cellophane soaked in glycerol-methylene blue solution is used to cover the remaining sample. The slides should be kept at room temperature or in an incubator at $40^{\circ} \mathrm{C}$ (except for hookworms) to clear the fecal debris. Microscopic examination can best be performed after 24-48 hours. The helminthic eggs per gram of stool $(E P G)$ is calculated as: No. of eggs in smear $\times 20$ (for $50 \mathrm{mg}$ template) to measure the infection intensity. ${ }^{17}$

T. trichiura, A. lumbricoides and Schistosoma eggs are easily detected and remain recognizable for months by the Kato-Katz method but it has a poor performance for the detection of hookworm infection since these eggs tend to disintegrate early (within $60 \mathrm{~min}$ ) resulting in falsenegative results. ${ }^{18}$ Moreover, S. stercoralis larvae also remain undetected. Although Kato-Katz is cost-effective and easy to perform, samples with low infection intensity and variable clearing/disintegration times may impede the concurrent detection of different STHs. ${ }^{16}$ A summary of representative studies evaluating the performance of KatoKatz with other microscopy based methods is provided in Table $1{ }^{18-29}$

\section{Stool Parasite Concentration Methods}

Microscopic detection of parasites can be improved by employing various techniques that concentrate the parasite in the sample and removes the fecal debris. These techniques are based on either the principle of flotation or centrifugal sedimentation.

\section{Centrifugation Based Technologies}

Formol ether or ethyl acetate concentration (FEC) method can be used for both fresh and preserved samples and allows recovery of parasites by centrifugation into a fecal pellet. Briefly, a small quantity of stool sample is allowed to pass through two layers of gauze into a conical tube containing $0.85 \%$ saline or $10 \%$ formalin and then mixed. Ethyl acetate is added to the fecal suspension for extraction of fat and debris and the tube is centrifuged $(500 \mathrm{~g}$ for a minimum of $10 \mathrm{~min}$ ) leaving the parasites in the sediment. The supernatant is decanted and the sediment is resuspended with saline or $10 \%$ formalin and examined as a wet preparation using 10x and 40x objectives, with or without iodine. Diluted formalin or sodium acetate-acetic acid-formalin (SAF) is used to inactivate the organisms and minimizes the risk of laboratory-acquired infection from feces. FEC stool samples can be examined in several 


\begin{tabular}{|c|c|c|c|c|}
\hline Helminth & $\begin{array}{c}\text { Bile } \\
\text { staining }\end{array}$ & Morphology & Comments & Microscopy \\
\hline $\begin{array}{l}\text { Ascaris lumbri- } \\
\text { coides } \\
\quad \text { Fertilized egg }\end{array}$ & $\begin{array}{l}\text { Bile } \\
\text { stained }\end{array}$ & $\begin{array}{l}\text { Size: } 45-75 \times 30-35 \\
\mu \mathrm{m}, \text { thick outer shell } \\
\text { with a prominent exter- } \\
\text { nal mammillated layer }\end{array}$ & $\begin{array}{l}\text { In decorticated eggs, the out- } \\
\text { er mamillated layer is absent }\end{array}$ & \\
\hline Unfertilized egg & $\begin{array}{l}\text { Bile } \\
\text { stained }\end{array}$ & $\begin{array}{l}\text { Size: } 85-95 \times 43- \\
47 \mu \mathrm{m}, \text { thinner shell } \\
\text { and variable mammil- } \\
\text { lated layer }\end{array}$ & & \\
\hline $\begin{array}{l}\text { Hook worm egg } \\
\text { (Ancylostoma and } \\
\text { Necator) }\end{array}$ & $\begin{array}{l}\text { Not Bile } \\
\text { stained }\end{array}$ & $\begin{array}{l}\text { Size: } 60-75 \times 35-40 \\
\mu \mathrm{m}, \text { Thin-shelled, col- } \\
\text { orless }\end{array}$ & $\begin{array}{l}\text { Prolonged standing }(>24 \mathrm{~h}) \text {, } \\
\text { larvae may hatch out of } \\
\text { eggs; these to be differentiat- } \\
\text { ed from } S \text {. stercoralis larvae }\end{array}$ & \\
\hline $\begin{array}{l}\text { Trichuris } \\
\text { trichiura egg }\end{array}$ & $\begin{array}{l}\text { Not Bile } \\
\text { stained }\end{array}$ & $\begin{array}{l}\text { Size: } 50-55 \times 20-25 \\
\mu \mathrm{m} \text {, thick-shelled bar- } \\
\text { rel-shaped, bipolar } \\
\text { "mucus plugs" }\end{array}$ & $\begin{array}{l}\text { Eosinophils and Charcot- } \\
\text { Leyden crystals may be pre- } \\
\text { sent in stool specimen }\end{array}$ & \\
\hline $\begin{array}{l}\text { Strongyloides ster- } \\
\text { coralis larvae }\end{array}$ & $\begin{array}{l}\text { Not Bile } \\
\text { stained }\end{array}$ & $\begin{array}{l}\text { Unsheathed rhabditi- } \\
\text { form larvae measuring } \\
200-300 \mu \mathrm{m} \times 16 \mu \mathrm{m}\end{array}$ & $\begin{array}{l}\text { Bulbed esophagus, short } \\
\text { buccal cavity differentiates it } \\
\text { from hookworm larva }\end{array}$ & $\underset{\text { Hotamom }}{\text { U }}$ \\
\hline
\end{tabular}

Figure 2 Summary of direct microscopy findings in different STH.

Notes: Images adapted from https://www.cdc.gov/dpdx/diagnosticprocedures/stool/morphcomp.html. Source: Centres for disease control and prevention [CDC], DPDx.

hours or days after the sample is preserved in formalin. However, this method has deficiencies due to the use of ether which is explosive, irritates the respiratory tract and may be harmful to laboratory personnel in addition to being time-consuming. ${ }^{16}$ Nikolay et al. (2014) have performed a meta-analysis of sensitivity of various diagnostic techniques for STH in the absence of a true gold standard. ${ }^{30} \mathrm{~A}$ few studies that have compared various microscopic techniques to Kato-Katz are presented in Table 1.

Recently, a closed, single-use Parasep ${ }^{\circledR}$ SF fecal parasite concentrator method has been developed by DiaSys Europe. This removes the sample debris and fat by a two-stage filtration matrix. This does not use any potentially harmful chemical agents and besides is user friendly, time-saving and cost-effective for diagnosis of helminth infections in large-scale epidemiological surveys. ${ }^{31} \mathrm{~A}$ modification of this method, the Mini Parasep ${ }^{\circledR}$ SF fecal parasite concentrator has been developed. Although this modification has been evaluated extensively for protozoal infections, its performance for STH diagnosis is reported in only a handful of studies. ${ }^{29-32}$ A clearer background with less fecal debris and a better yield of T. trichiura has been noted with Mini Parasep ${ }^{\circledR} \cdot{ }^{29,31-34}$ Additionally, the mean time for processing is much faster with Parasep ${ }^{\circledR}$ than the conventional formol-ether-acetate concentration (4-5 min vs 10-15 min/sample), which is a significant time-saver. 


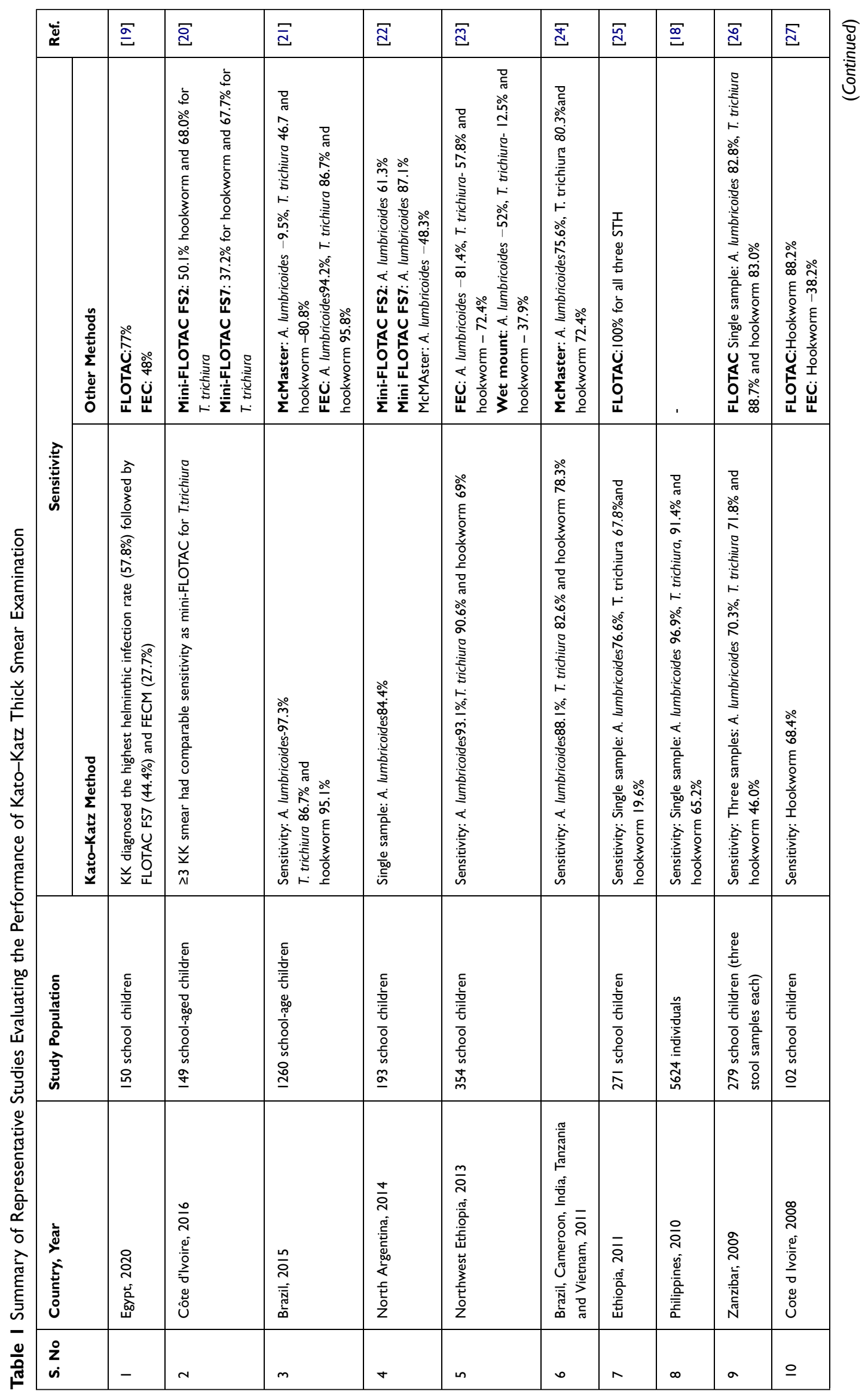




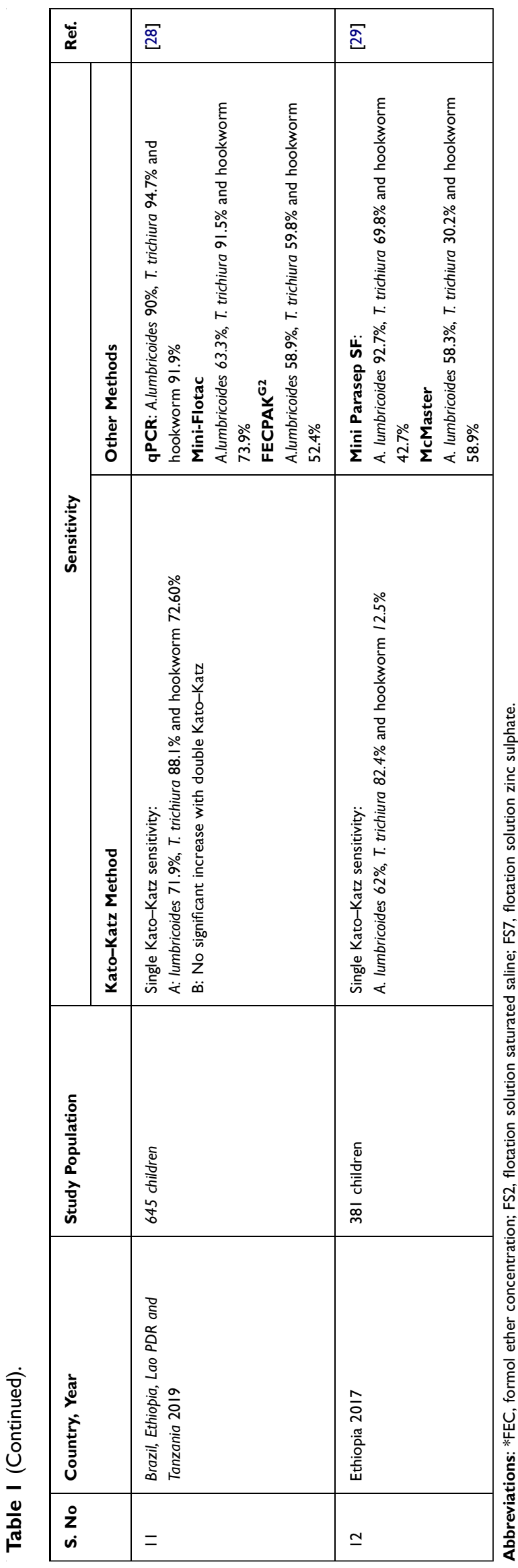

However, conventional FEC costs less than Parasep ${ }^{\circledR}$ (0.30 vs 0.9 USD /sample). ${ }^{33}$ Higher sensitivity of Mini Parasep $^{\circledR}$ SF has been reported for A. lumbricoides, Kato-Katz for T. trichiura and McMaster for hookworm eggs. ${ }^{34}$

\section{Flotation-Based Technologies}

Most parasite eggs have a specific gravity of 1.05-1.20 which allows them to float when the stool sample is mixed in a high specific gravity solution (range 1.181.27). The commonly used flotation solutions are zinc sulphate or brine solution. Successor technologies of the Cornell Wisconsin centrifugal flotation method, namely McMaster method and FLOTAC/Mini-FLOTAC, have shown promise. ${ }^{17,28,35,36}$

\section{McMaster Technique}

This technique uses a counting chamber which enables a known volume of fecal suspension $(2 \times 0.15 \mathrm{~mL})$ to be examined microscopically. The McMaster chamber has two compartments, each with a grid etched onto the upper surface. When filled with a suspension of feces in flotation fluid, much of the debris sinks while the eggs float to the surface under the grid, where they can easily be seen and counted relatively quickly. With a known weight of feces and volume of flotation fluid used to prepare the suspension, the EPG count can easily be derived by multiplying the number of eggs under the marked areas by a simple conversion factor. ${ }^{21}$ The McMaster method is inexpensive, easy and has been extensively used in veterinary parasitology and even in human studies for estimation of anthelmintic cure rates. ${ }^{35}$ The overall diagnostic performances of the McMaster method and Kato-Katz is comparable for STHs according to a recent meta-analysis. ${ }^{30}$ In a study by Periago et al., the Kato-Katz outperformed the McMaster method for all three STHs, especially for unfertilized A. lumbricoides egg detection. ${ }^{21}$ The reason for the poor performance of the flotation methods for detection of the unfertilized Ascaris eggs is because of their high specific gravity $(\mathrm{SG}=1.18$ ) and absence of lipoidal membrane that prevents the equilibration of the egg with the external flotation media causing the egg to sink instead of float in the saline. ${ }^{21,22,36}$ This deficiency and the need for a special counting chamber are disadvantages of this technique. 


\section{FLOTAC}

Centrifugal flotation is the basis of the FLOTAC technology and it consists of a cylindrical device with two flotation chambers of $5 \mathrm{~mL}$ capacity allowing analysis of more fecal material compared with Kato-Katz (100 mg vs $42 \mathrm{mg}) .{ }^{37-39}$ It has a high sensitivity with the additional advantage of being a closed system that protects the operators, preserves the sample and even allows repeated subsequent sample examinations. However, the methodological complexity and the need for a specific device for sample centrifugation may limit its applicability in laboratories with resource constraints. Mini-FLOTAC is a simplified version of this device without the centrifugation step. $^{20,22,37-39}$ A recent meta-analysis revealed a sensitivity of approx. $92.7 \%$ for FLOTAC vs $42.8 \%$ for direct microscopy. ${ }^{30}$ The Kato-Katz method had a sensitivity of $74-95 \%$ for detection of all STH in high infection intensity, which drops to $53-80 \%$ in settings of low infection intensity, with the poorest performance for A. lumbricoides and hookworms. The FLOTAC method has the highest sensitivity in settings of both low and high infection intensity; however, the average egg counts are significantly lower compared with the Kato-Katz method. ${ }^{25,26,30}$ Therefore, validation of this method in varying epidemiological settings with different intensity of infections is needed before recommendation for largescale community use.

\section{Digitized Microscopy}

To ensure the objective evaluation of current technologies and prioritization of products for use in STH control programs, a detailed framework has been formulated to link the program decision points with analogous target product profiles (TPPs). ${ }^{40}$ Three opportunities for improvement in STH diagnosis were suggested: "First, integration of quality control/assurance for sample processing and egg counting; second, increasing the sample throughput, and; thirdly, internet connectivity to make test results accessible for remote interpretation." ${ }^{, 40}$ These technical requirements are met by $\mathrm{FECPAK}^{\mathrm{G} 2}$, a tool developed for helminth eggs counting in fecal samples from sheep, cattle, horses, camels etc. ${ }^{40}$ In FECPAK, stool sample dissolved in flotation solution is filled in a tube with a central pillar and the eggs are allowed to concentrate into one microscopic field of view. Then an attachment takes images of the eggs that have accumulated into a single viewing area and this digital image is saved and uploaded online. ${ }^{41}$ In a study by Moser et al. a sensitivity of $75.6 \%, 71.5 \%$ and $65.8 \%$ for A. lumbricoides, hookworm and T. trichiura detection, respectively, was observed for FECPAK $^{\mathrm{G} 2}$ which was much lower than the Kato-Katz method. ${ }^{42}$ However, with rigorous development, this could be an interesting epidemiological and diagnostic tool.

\section{Lab-On-A-Disk Technology}

Another technique with compliance to the TTPs is the lab-on -a-disk (LOD) technology. This is a microfluidic platform based on both the centrifugation and flotation of eggs for concentration within an imaging zone, followed by image capture. ${ }^{43}$ This technique holds potential for point-of-care application, however, adequate training and education of the users and quality management aspects in terms of device calibration and maintenance must be ensured. A field evaluation of the LOD technology in human samples from Ethiopia revealed successful capture of STH eggs in the image viewing zone of the LOD device even in low infection intensity settings. ${ }^{43}$ An almost perfect agreement with Mini-FLOTAC (Kappa score: 0.91) was seen; in fact, more eggs were counted in the LOD technique.

\section{Culture-Based Methods}

The use of parasite culture in STH infections is seldom the mainstay for diagnosis and is primarily applied in research settings, except for the recovery of S. stercoralis. ${ }^{4-46}$ However, great care must be taken since larvae are infective and can be a biohazard for lab personnel. A summary of various larval detection methods used are shown in Table 2.

\section{Serology Based Methods Antibody Detection}

Antibody detection in serum is not particularly useful for STH diagnosis except for $S$. stercoralis. Although indirect immunofluorescence microscopy (IFAT) and gelatin particle agglutination (GPAT) test have high sensitivity (8198\%) and specificity (74\%), the requirement for a large amount of living, infective larvae limit their use. ${ }^{44,47}$ Immunoblot analysis using antigens of $S$. stercoralis and S. ratti was used in a study in 2003 to overcome this drawback and had shown sensitivity ranging from 65 $100 \%{ }^{48}$ However, there are no further reports on evaluation of these tests and therefore their validity is questionable. 
Table 2 Characteristics of Larval Detection Methods for the Diagnosis of Strongyloidiasis or Hookworm Infection

\begin{tabular}{|c|c|c|c|}
\hline Method & Procedure & Comments & Reference \\
\hline Baermann method & $\begin{array}{l}\text { A large amount of feces }(10 \mathrm{~g}) \text { are kept with warm water over } \\
\text { gauze in a glass funnel. Soil samples can also be used } \\
\text { Larvae crawl out of the feces and migrate into the water after } \\
\mathrm{I}-2 \mathrm{~h} \\
\text { Centrifuged water sediment is monitored for larvae of } \\
\text { hookworms or Strongyloides by microscopy }\end{array}$ & $\begin{array}{l}\text { - Almost } 4 \text { times higher sensitivity than } \\
\text { FEC or DS } \\
\text {-Requires fresh and non-refrigerated stool } \\
\text { samples } \\
\text { - Soil samples can also be used to detect } \\
\text { larvae } \\
\text { - Time-consuming, labor-intensive and } \\
\text { a large quantity of stool is required }\end{array}$ & [44] \\
\hline $\begin{array}{l}\text { Harada-Mori filter } \\
\text { paper strip culture }\end{array}$ & $\begin{array}{l}\text { A small amount of stool sample }(0.5-1.0 \mathrm{~g}) \text { is spotted on filter } \\
\text { paper strips, inserted into a test tube with water slightly below } \\
\text { the fecal spot and incubated upright at } 25 \text { to } 28{ }^{\circ} \mathrm{C} \text { for up to } 10 \\
\text { days } \\
\text { Test tube fluid is monitored for larvae of hookworms or } \\
\text { Strongyloides by microscopy daily }\end{array}$ & $\begin{array}{l}\text { - More sensitive compared with FEC or DS } \\
\text { but less sensitive than Baermann or agar } \\
\text { plate methods } \\
\text {-Refrigerated or preserved specimen } \\
\text { cannot be used }\end{array}$ & [45] \\
\hline $\begin{array}{l}\text { Filter paper/slant } \\
\text { culture technique } \\
\text { (Petri dish) }\end{array}$ & $\begin{array}{l}\text { A slide containing fresh stool }(\mathrm{I}-2 \mathrm{~g}) \text { is placed in a glass or } \\
\text { plastic Petri dish with water } \\
\text { Petri dish fluid is monitored for larvae of hookworms or } \\
\text { Strongyloides by microscopy daily up to } 10 \text { days }\end{array}$ & $\begin{array}{l}\text {-Low sensitivity } \\
\text {-Refrigerated or preserved specimen } \\
\text { cannot be used }\end{array}$ & [45] \\
\hline Charcoal culture & $\begin{array}{l}\text { Stool suspension in water and ground charcoal (both } 20-40 \mathrm{~g} \text { ) } \\
\text { are mixed and placed in a storage dish and incubated for 5-6 } \\
\text { days } \\
\text { Sediments of centrifuged water are monitored for egg hatching } \\
\text { and/or larvae development }\end{array}$ & $\begin{array}{l}\text { - Suitable in remote regions } \\
\text { - Long incubation period } \\
\text {-An efficient method to harvest large } \\
\text { amounts of larvae for experimental work }\end{array}$ & [44] \\
\hline $\begin{array}{l}\text { Koga Agar Plate } \\
\text { culture }\end{array}$ & $\begin{array}{l}\text { Feces }(3 \mathrm{~g}) \text { are placed on an agar plate with double walls (with } \\
\text { an outer surrounding solution of glycerin) and incubated ( } 2-6 \\
\text { days) } \\
\text { Larvae form tracks on agar surface and are visualized by } \\
\text { microscopy }\end{array}$ & $\begin{array}{l}\text { - More sensitive than filter paper culture, } \\
\text { DS or FEC } \\
\text {-Strong diagnostic agreement with } \\
\text { Baermann's method } \\
\text { - Time-consuming }\end{array}$ & [46] \\
\hline
\end{tabular}

Notes: Caution must be exercised and gloves must be worn as larvae may be infective and can penetrate through skin.

Abbreviations: FEC, formol ether concentration; DS, direct smear.

The crude extracts of filariform larvae, from S. stercoralis/S. venezuelensis or $S$. ratti have been used in several in-house Enzyme-linked Immunosorbent Assays (ELISAs) and commercial kits with sensitivity ranging from $73-100 \%{ }^{44}$ however, cross-reactivity with other nematodes, especially filarial worms, and poor performance in immunosuppressed individuals are observed. ${ }^{49}$ The falsepositive results in infections due to Echinococcus and Toxocara are less commonly seen with ELISA compared with IFAT. It is worth mentioning that the ELISA-based studies have been performed in case-control study designs using serum samples from patients with known strongyloidiasis compared with healthy controls and not with individuals harbouring other nematode infections, causing falsely high estimates of performance. Another concern is the overestimation of disease burden by serology versus underestimation by parasitological techniques since stool examination by itself is an imperfect gold standard. Although increased specificity of $13.3 \%$ on substitution of IgG with IgG4 in Strongyloides ELISA is seen, very few studies have focused on detection of other antibody isotypes. ${ }^{50}$

The luciferase immunoprecipitation system (LIPS) has been developed using a recombinant Strongyloides antigen (NIE) ${ }^{51,52}$ This is a rapid test $(<3$ hours) and has a good sensitivity $(97 \%)$ and specificity $(100 \%)$ with no crossreaction. $^{52}$ Additionally, it uses recombinant antigen, enabling mass production and purification compared with the time-consuming preparation of crude antigen which depends on the collection of stool from infected humans/ animals. Detection of IgG and IgG4 specific antibodies by using the luciferase-tagged recombinant Ss-NIE-1 protein of 
S. stercoralis by LIPS has shown a sensitivity and specificity of $93 \%$ and $95 \%$, respectively. ${ }^{53}$ Recently, a dipstick lateral flow assay using recombinant NIE (rNIE) and recombinant Ssla (rSs1a) proteins was developed. It demonstrated a sensitivity of $91.3 \%$ and $100 \%$ specificity for serodiagnosis of strongyloidiasis and merits further evaluation. ${ }^{54}$

\section{Coproantigen Detection}

Coproantigen detection could have the potential to be used in point-of-care immunoassay-based tests such as that for malaria. The detection of coproantigen of $S$. stercoralis by ELISA was reported to have good sensitivity and specificity but no further results of its evaluation are available. ${ }^{55}$ Recently BA-1, an excretory-secretory product of A. lumbricoides, has been investigated as a coproantigen marker of infection by ELISA. ${ }^{56}$ The Ascaris BA-1 ELISA had a lower limit of quantification of $2.74 \mathrm{ng} / \mathrm{g}$ stool and its evaluation in a panel of stool samples from school-aged children residing in Kenya revealed a sensitivity and specificity of $91.5 \%$ and $95.3 \%$, respectively. Apart from the qualitative assessment of infection, quantitative reflection into the intensity of infection was also seen and a value of $18.2 \mathrm{ng} / \mathrm{g}$ stool of the ABA-1 was found to be the cut-off between low and moderate intensities of infection. It was also seen that the levels of ABA-1 strongly decreased after treatment. A commercial kit for the detection of Ascaris, hookworms and Trichuris antigens has been patented and marketed by IDEXX laboratories for veterinary use. ${ }^{57}$

Recently, urinary biomarkers of $A$. lumbricoides infection, such as 2-methyl butyramide, 2-methyl pentanoyl carnitine (2-MPC) and 2-methyl-valeramide metabolites have also been evaluated. ${ }^{58,59}$ Of these, 2-MPC has been found to have an accuracy of $85.7 \%$ for detection of infection with better performance (90.5\% accuracy) for moderate to heavy infection. ${ }^{58}$ A substantial reduction after albendazole treatment was also seen with a significant association between urine 2-MPC levels and worm/egg counts. ${ }^{58}$ However, the same authors did not find this biomarker in infected Indonesian subjects. ${ }^{59}$

\section{Molecular Methods}

Over the past decade, efforts to develop and evaluate molecular diagnostics for STH have gained momentum for epidemiologic studies to guide interventional strategies and diagnostics in travelers returning from endemic areas. ${ }^{60}$ However, there is still a dearth of molecular platforms for the detection of STH. Part of the difficulty in estimation of the performance of molecular tests is the lack of an optimum gold standard, as generally the stool-based microscopic examination methods are used as reference standard albeit having a low sensitivity. There are still no clear cut relevant criteria to validate laboratory developed PCRs. The diagnostic reliability of molecular methods also depends on the efficiency of DNA extraction and sample preservation before sample testing. There are a lot of inhibitors in the stool samples that affect the sensitivity of PCR. Moreover, preservation of samples in formalin also inhibits the PCR. It should be noted that helminthic eggs, especially those of T. trichiura, are more difficult to break thus affecting the DNA extraction efficiency and PCR. ${ }^{60,61}$ Ayana et al. (2019) compared four DNA extraction and preservation methods for the molecular detection and quantification of soil-transmitted helminths in fecal samples and found significant differences in DNA recovery ${ }^{60}$. However, if the samples had been kept in the preservatives, there was no loss of sensitivity attributed to delay in DNA extraction which makes it suitable for collection of samples in preservatives from remote areas and their subsequent analysis at a distant laboratory. ${ }^{60}$

Great strides in the sequencing of nematode genomes with the availability of nematode sequence data have facilitated the discovery and development of assays based on more sensitive and species-specific genomic targets. ${ }^{61,62}$ Ribosomal sequences and mitochondrial targets such as cytochrome oxidase are found in most eukaryotic cells making them attractive target choices. ${ }^{62}$ In STH, a large portion of the genome is comprised of repetitive non-coding DNA elements, which are useful PCR targets for STH. ${ }^{63}$ It should be noted that some types of repetitive DNA, such as simple short nucleotide repeats or sequence variation within the repeat, impact the suitability of repeat-based molecular assays. ${ }^{63,64}$ The repeats can be variable-type (i.e., copy number is not consistent within species) or constant-type (copy number is consistent within species) and understanding this variation on a species-byspecies basis and its impact on the sensitivity of any molecular assay is needed. ${ }^{64}$ New target development for repeat-based qPCR assays is easier using new bioinformatic tools such as RepeatExplorer, TAREAN etc. which can help in amplifying as little as 20 ag (attogram) of genomic DNA. ${ }^{63}$

The gene targets used in PCR to detect STH are shown in Table 3.

The main advantage of PCR-based assays lies in their higher sensitivity and the ability to multiplex for detecting concurrent infections, although singleplex PCR is slightly more sensitive compared with multiplexed assays. ${ }^{65,66}$ Use of quantitative PCR (qPCR) provides even more accurate prevalence estimates compared with microscopy and is amenable to 
Table 3 Gene Targets Used for the Molecular Diagnosis of Soil Transmitted Helminths

\begin{tabular}{|l|l|l|}
\hline Gene Target & STH & Primer Reference \\
\hline ITS-I & $\begin{array}{l}\text { Strongyloides stercoralis, } \\
\text { Ascaris lumbricoides, Trichuris trichiura }\end{array}$ & {$[61,65-68]$} \\
\hline ITS-2 & Ancylostoma duodenale/ceylanicum, Necator americanus, Trichuris trichiura & {$[64]$} \\
\hline I8S & Strongyloides stercoralis & {$[67]$} \\
\hline Cytochrome oxidase I & Strongyloides stercoralis, A. duodenale, Ascaris lumbricoides, Necator americanus & {$[62,65]$} \\
\hline Sequence repeats & Strongyloides stercoralis, A. duodenale, Necator americanus, Trichuris trichiura & {$[65]$} \\
\hline ITS-I, 5.8S, ITS-2 & Strongyloides stercoralis & {$[64,69]$} \\
\hline
\end{tabular}

Abbreviations: ITS, internal transcribed spacer; STH, soil-transmitted helminth.

multiplexing. ${ }^{69,70}$ Moreover, since the frequency of mass drug treatment of a population is determined by the prevalence of disease in the community, which in turn is greatly dependent on the sensitivity of the diagnostic test used, quantitative PCR has a greater role to play for accurate estimates and also after completion of MDA, when it is critical to define a threshold under which recrudescence of disease can occur. ${ }^{69}$ However, qPCR can be technically demanding, complex and expensive and there is lack of standardization across different laboratories. Further, it is not very straightforward to correlate copies of molecular target to egg numbers in feces and worm numbers in gut, as number of copies of that target and difference of ploidy among unfertilized and fertilized eggs varies. ${ }^{69}$ These hurdles are increasingly being removed and there are efforts to commercialize these tests.

In a recent study to evaluate the diagnostic performance of Kato-Katz, Mini-FLOTAC, FECPAK G2 and qPCR, all diagnostic techniques showed a sensitivity of $\geq 90 \%$ for all of the moderate-to-heavy intensity STH infections. However, only qPCR had a sensitivity that was superior to a single Kato-Katz for all STHs or infections of very low intensity. For qPCR, there was a positive significant correlation between the egg counts of a single Kato-Katz and the DNA concentration. qPCR is the only method that could be considered in the phase that aims to seek confirmation for cessation of program. ${ }^{35}$ In addition, pooling of samples has been tested as a strategy to reduce cost and time and found to be costeffective, time and labour saving without loss of sensitivity or accuracy. $^{71}$

A newer molecular tool, Loop-mediated amplification assay (LAMP) is a single-step method amplifying target DNA sequences isothermally. ${ }^{72-76}$ It only requires a heat block or incubator/water bath and is more tolerant of biological inhibition. For improving sensitivity, fluorescent probes, DNA-functionalized gold nanoparticles etc. can be used. However, due to difficulties in primer design, false positives and carry over-cross contamination, further validation of LAMP assays is needed. Recently, a colorimetric isothermal assay using SmartAmp2 primer was developed to identify STH by targeting the amplification of a specific sequence of the $\beta$-tubulin gene visualized by hydroxy naphthol blue (HNB) dye in LAMP format. ${ }^{73}$ The unique asymmetrical primer design makes this a very specific assay with easy visual result inspection, empowering its development as a portable tool for field surveys.

\section{Other Aids in Clinical Diagnosis}

Low hemoglobin, low ferritin and eosinophilia $(>600 \mu \mathrm{L}$ or $>6 \%$ of the thin-layer chromatography) can be used as indirect indicators of STH infection. ${ }^{16}$ Occult blood in stool has been used as a surrogate marker of chronic hookworm infection. ${ }^{77}$ In ascariasis, Charcot-Leyden crystals and eosinophils may be found in the sputum. In the case of $S$. stercoralis, duodenal material may be examined for the presence of larvae using the string test (or entero-test capsule). ${ }^{44}$ The use of other investigations such as diagnostic radiology, histopathology, and intradermal skin tests have also been reported but are seldom used routinely. ${ }^{16}$

\section{Choosing the Right Diagnostic Technique}

Despite considerable progress to control STH over several decades, we are still far from identifying a fully adequate diagnostic test. In general, the consensus is still to strive towards the development of novel sensitive technologies or further optimize the current methods. To choose from the 
present armamentarium of diagnostic assays, a few aspects which merit consideration are the diagnostic performance, cost-effectiveness, ease of performance, rapidity and in-field applicability. Apart from the performance of the technique per se, adequate training and stringent quality-control/qualityassurance measures greatly improve the performance of any diagnostic assay. Importantly, different phases of STH control programs would require different techniques; microscopy would suffice to map prevalence in a geographic area but more sensitive molecular tests will be required for post MDA surveillance and to measure success of control programs.

\section{Detection of STH in Environmental Samples}

The detection of STH eggs and quantification in other sample types such as wastewater, sludge, plants, etc., is also important for control of these infections. Unfortunately, due to differences in the moisture content and amount of solids or soil particles etc. in the environmental samples, there is significant heterogeneity in the detection of STHs from these samples. ${ }^{78}$ The conventional microscopic techniques are largely insensitive, time-consuming and labor intensive for environmental samples. Thus, various modifications of traditional methods and newer molecular technologies are now being increasingly applied. Due to dilution in the environment, helminth egg concentrations in wastewater are normally very low, necessitating the use of large volumes (1-10 L) for detection. The major hurdle for the molecular detection of STH eggs is the extraction of the nucleic acid of good quantity and quality due to the hardy eggshells, and high amounts of suspended solids or PCR inhibitors in most of the samples. A high concentration of STH may be seen in sludge, biosolids and compost since eggs attach to particulate material, and thus usually around $2-5 \mathrm{~g}$ dry weight of sludge is sampled. An adequate amount of soil sample varies from 10 $50 \mathrm{~g}$ of dry soil, mostly from the top $0-5 \mathrm{~cm}$ layer. Higher egg recovery and less variation in egg count are observed in sand than from clay and silt. ${ }^{80}$ For vegetable samples, the preferred weight is 100-250 g (lower for grass). It should be noted, however, that even with the same volumes of sample, the concentration of helminth eggs differs regionally depending on the local prevalence of infection and solid content in the sample. ${ }^{78}$ Also, it is important to determine the number of viable STH eggs for risk estimation per international guidelines. ${ }^{78-81}$ A schematic diagram depicting the steps involved in the detection of STH from environmental samples is shown in Figure 3.
Briefly, the samples need to be homogenized first to break down coarse material into finer particles, followed by separation of eggs from particles using different solutions (mainly anionic detergents). Then the samples are filtered, thereby allowing eggs to pass through for further analysis. The samples are concentrated by sedimentation or flotation. Phase extraction to remove lipid/ether soluble material from samples may be performed after flotation using ethyl acetate and diethyl ether (for lipophilic extraction) and sulphuric acid and acetoacetic acid buffer (for hydrophilic extraction). The exposure time should be minimal to reduce the adverse impact of chemicals on egg morphology and recovery.

After this, the viable STH eggs per sample are assessed. The viability assessment can be done by incubation for larval development, which is time-consuming and is subject to observer expertise. Viable and non-viable eggs can also be differentiated by using vital stains. Another emerging and rapid method is based on differential integrity of viable and non-viable cell membranes by using the BacLight LIVE/DEAD Viability Kit that was initially developed for the enumeration of viable bacteria. $^{82}$

Recently, image analysis software or systems have been developed to identify and quantify helminth eggs in wastewater using various image processing tools and pattern recognition algorithms. ${ }^{82}$ These have a reported sensitivity of $80-90 \%$ and a specificity of $99 \%$ depending on the total suspended solids content of the wastewater samples and the time taken to examine each sample is less than a minute. It should be remembered that even when such software is used, the eventual identification still depends on proper sample processing. Improving the optics with provision for better quality images could potentially make use of smartphones by enabling the consultation of remotely located experts to interpret the tests, especially for surveys in remote and low-income areas.

Molecular methods such as real-time PCR (qPCR), digital/ droplet PCR (ddPCR) etc. also allow the accurate detection of STH eggs in environmental samples. ${ }^{76}$ The application of ddPCR in poultry water samples revealed a better performance compared with qPCR and culturebased methods for the detection of zoonotic pathogens. ${ }^{82,83}$ However, further research on the optimization of chip-based PCR or ddPCR in environmental samples is warranted. Another approach is flow cytometry, which is based on the differentiation of eggs based on sizes. ${ }^{78}$ In fact, fluorescent 


\section{Sample collection}

Wastewater/ water, vegetables and plants, soil

\section{Homogenization}

By blending or mixing

\section{Separation}

To separate eggs from particles treatment with:

- Detergents: Tween 80, Triton-X, 1\% 7X (best), Ammonium

bicarbonate (if more soil)

- For water: Saline, PBS, Tweem 80, water

\section{Filtration}

Filter through sieve of $32-36$ micon size (not for vegetable samples)

\section{Concentration}

$\quad$ Sedimentation
Passive sedimentation in
open, straight sided contained
of $10 \mathrm{~L}$ volume for 1 hour

of 10L volume for 1 hour

\section{Floatation *}

Using solution with $>1.25 \mathrm{sp}$. Gravity (ZnSO4, MgSO4, Sodium nitrate, sucrose, sodium dichromate

\section{Viability determination}

-Incubation fin sulphuric acid/ formalin at $22-26^{\circ} \mathrm{C}$ for $21-$ 30 days for larvae development

-Stain with lugol's iodine, Safranine O, eosin Y

-BacLight LIVE/DEAD Bacterial Viability Kit

\section{Molecular tests}

Figure 3 A schematic diagram depicting the steps involved in the detection of STH from environmental samples. *phase extraction may be performed after flotation step.

staining may even enable us to determine the stage of egg development or viability. However, the cost of equipment and the need for trained staff may cause hindrance in the application of this technique.

\section{Conclusion}

STH infections have a substantial socio-economic impact and entail huge physical and developmental consequences for infected populations. The microscopic examination of stool 
is an acceptable measure for the assessment of infection levels in high-intensity endemic areas. The sensitivity of microscopy-based techniques can be improved greatly by properly training the involved laboratory staff. However, the major challenge of conventional techniques is that they are timeconsuming and involve cumbersome procedures in sample analysis. Less cumbersome and more sensitive FLOTAC techniques may be useful in such areas. However, in areas of low endemicity, or during the elimination phase, the relevance of microscopy-based methods is limited and more sensitive molecular techniques such as PCR may be needed. qPCR with its ability to provide estimation of intensity of infection has been demonstrated to be of potential, especially when cessation of intervention by mass drug administration is considered or for surveillance of STH in low endemic areas or travelers returning from endemic regions. Moreover, the molecular techniques are important to detect new/emerging species. However, more rigorous studies are required to accurately relate qPCR data to worm burden.

\section{Future Perspective}

The development of new diagnostic methods with broadspectrum applicability in different sample matrices is imperative for STH detection. Although the development of molecular tools has numerous advantages in the context of sensitivity, ease of performance, reproducibility and automation, they still have many shortfalls; the most important being high cost which limits applicability in regions where sophisticated equipment and adequate user training is not possible. However, pooling of samples and cost-effective modifications of existing molecular methods are being attempted to circumvent problems associated with PCR.

There may be a potential for antibody/ antigen detection but these need to be evaluated in the field. Development of new techniques, digitization/automation of procedures, implementation of clear protocols, identification of novel biomarkers and multiplexing hold promise for STH diagnosis. Additionally, rigorous multi-centric validation of new diagnostic assays, particularly in low endemic settings, is imperative.

\section{Disclosure}

The authors report no conflicts of interest in this work.

\section{References}

1. Soil-transmitted helminth infections. Available from: https:/www. who.int/news-room/fact-sheets/detail/soil-transmitted-helminthinfections. Accessed December 16, 2020.
2. Colella V, Bradbury R, Traub R. Ancylostoma ceylanicum. Trends Parasitol. 2021;25:S1471-4922. doi:10.1016/j.pt.2021.04.013

3. Hotez PJ, Alvarado M, Basáñez MG, et al. The Global Burden of Disease Study 2010: interpretation and Implications for the Neglected Tropical Diseases. PLoS Negl Trop Dis. 2014:8. doi:10.1371/journal. pntd.0002865

4. Hay SI, Abajobir AA, Abate KH, et al. Global, regional, and national disability-adjusted life-years (DALYs) for 333 diseases and injuries and healthy life expectancy (HALE) for 195 countries and territories, 19902016: a systematic analysis for the Global Burden of Disease Study 2016. Lancet. 2017;390:1260-1344. doi:10.1016/S0140-6736(17)32130

5. Intestinal nematode infections - level 3 cause | institute for Health Metrics and Evaluation n.d. Available from: http://www.healthdata. org/results/gbd_summaries/2019/intestinal-nematode-infections-level -3-cause. Accessed January 18, 2021.

6. Kuong K, Fiorentino M, Perignon M, et al. Cognitive performance and iron status are negatively associated with hookworm infection in Cambodian schoolchildren. Am J Trop Med Hyg. 2016;95:856-863. doi:10.4269/ajtmh.15-0813

7. Clarke NE, Clements ACA, Doi SA, et al. Differential effect of mass deworming and targeted deworming for soil-transmitted helminth control in children: a systematic review and meta-analysis. Lancet. 2017;389:287-297. doi:10.1016/S0140-6736(16)32123-7

8. Pabalan N, Singian E, Tabangay L, Jarjanazi H, Boivin MJ, Ezeamama AE. Soil-transmitted helminth infection, loss of education and cognitive impairment in school-aged children: a systematic review and meta-analysis. PLoS Negl Trop Dis. 2018;12(1): e0005523. doi:10.1371/journal.pntd.0005523

9. Garrison A, Boivin M, Khoshnood B, et al. Soil-transmitted helminth infection in pregnancy and long-term child neurocognitive and behavioral development: a prospective mother-child cohort in Benin. PLoS Negl Trop Dis. 2021;15(3):e0009260. doi:10.1371/journal. pntd.0009260

10. Anderson R, Truscott J, Hollingsworth TD. The coverage and frequency of mass drug administration required to eliminate persistent transmission of soil-transmitted helminths. Philos Trans R Soc B Biol Sci. 2014;369(1645):20130435. doi:10.1098/rstb.2013.0435

11. Montresor A, Mupfasoni D, Mikhailov A, et al. The global progress of soil-transmitted helminthiases control in 2020 and World Health Organization targets for 2030. PLoS Negl Trop Dis. 2020;14: e0008505. doi:10.1371/journal.pntd.0008505

12. Hotez PJ, Bundy DAP, Beegle K, et al. Helminth Infections: soiltransmitted Helminth Infections and Schistosomiasis. In: Jamison DT, Breman JG, Measham AR, et al. editors. Disease Control Priorities in Developing Countries. 2nd;New York: Oxford University Press; 2006.

13. Keiser J, Utzinger J. Community-wide soil-transmitted helminth treatment is equity-effective. Lancet. 2019;393(10185):2011-2012. doi:10.1016/S0140-6736(18)32981-7

14. Amoah ID, Adegoke AA, Stenström TA. Soil-transmitted helminth infections associated with wastewater and sludge reuse: a review of current evidence. Trop Med Int Heal. 2018;23:692-703. doi:10.1111/ tmi. 13076

15. Khurana S, Sethi S. Laboratory diagnosis of soil transmitted helminthiasis. Trop Parasitol. 2017;7:86. doi:10.4103/TP.TP_29_17

16. Garcia LS, Arrowood M, Kokoskin E, et al. Laboratory diagnosis of parasites from the gastrointestinal tract. Clin Microbiol Rev. 2018;31: e00025-17. doi:10.1128/CMR.00025-17

17. World Health Organization. Bench Aids for the Diagnosis of Intestinal Parasites. second ed. Geneve: World Health Organization; 2019.

18. Tarafder MR, Carabin H, Joseph L, Balolong E, Olveda R, McGarvey ST. Estimating the sensitivity and specificity of KatoKatz stool examination technique for detection of hookworms, Ascaris lumbricoides and Trichuris trichiura infections in humans in the absence of a "gold standard.". Int $J$ Parasitol. 2010;40:399-404. doi:10.1016/j.ijpara.2009.09.003 
19. Allam AF, Farag HF, Lotfy W, Fawzy HH, Elhadad H, Shehab AY. Comparison among FLOTAC, Kato-Katz and Formalin Ether Concentration Techniques for Diagnosis of Intestinal Parasitic Infections in School Children in an Egyptian Rural Setting. Parasitology. 2020;148(3):289-294. doi:10.1017/S0031182 020001675

20. Coulibaly JT, Ouattara M, Becker SL, et al. Comparison of sensitivity and faecal egg counts of Mini-FLOTAC using fixed stool samples and Kato-Katz technique for the diagnosis of Schistosoma mansoni and soil-transmitted helminths. Acta Trop. 2016;164:107-116. doi:10.1016/j.actatropica.2016.08.024

21. Periago MV, Diniz RC, Pinto SA, et al. The right tool for the job: detection of soil-transmitted helminths in areas co-endemic for other helminths. PLoS Negl Trop Dis. 2015;9(8):e0003967. doi:10.1371/ journal.pntd.0003967

22. Barda B, Cajal P, Villagran E, et al. Mini-FLOTAC, Kato-Katz and McMaster: three methods, one goal; highlights from north Argentina. Parasit Vectors. 2014;7(1):271. doi:10.1186/1756-33057-271

23. Endris M, Tekeste Z, Lemma W, Kassu A. Comparison of the Kato-Katz, Wet Mount, and Formol-Ether Concentration Diagnostic Techniques for Intestinal Helminth Infections in Ethiopia. ISRN Parasitology. 2013;180439. doi:10.5402/2013/180439

24. Levecke B, Behnke JM, Ajjampur SSR, et al. A Comparison of the Sensitivity and Fecal Egg Counts of the McMaster Egg Counting and Kato-Katz Thick Smear Methods for Soil-Transmitted Helminths. PLoS Negl Trop Dis. 2011;5:e1201. doi:10.1371/journal.pntd.0001201

25. Habtamu K, Degarege A, Ye-Ebiyo Y, Erko B. Comparison of the Kato-Katz and FLOTAC techniques for the diagnosis of soil-transmitted helminth infections. Parasitol Int. 2011;60:398-402. doi:10.1016/j.parint.2011.06.020

26. Knopp S, Rinaldi L, Khamis IS, et al. A single FLOTAC is more sensitive than triplicate Kato-Katz for the diagnosis of low-intensity soil-transmitted helminth infections. Trans $R$ Soc Trop Med Hyg. 2009;103:347-354. doi:10.1016/j.trstmh.2008.11.013

27. Utzinger J, Rinaldi L, Lohourignon LK, et al. FLOTAC: a new sensitive technique for the diagnosis of hookworm infections in humans. Trans R Soc Trop Med Hyg. 2008;102:84-90. doi:10.1016/ j.trstmh.2007.09.009

28. Cools P, Vlaminck J, Albonico M, et al. Diagnostic performance of a single and duplicate Kato-Katz, Mini-FLOTAC, FECPAKG2 and qPCR for the detection and quantification of soil-transmitted helminths in three endemic countries. PLoS Negl Trop Dis. 2019:13. doi:10.1371/journal.pntd.0007446

29. Adugna S, Kebede T, Mekonnen Z, Degarege A, Liang S, Erko B. Diagnostic performance of Mini Parasep ${ }^{\mathbb{B}}$ solvent-free faecal parasite concentrator relative to Kato-Katz and McMaster for the diagnosis of intestinal parasitic infections. Trans $R$ Soc Trop Med Hyg. 2017;111:572-578. doi:10.1093/trstmh/try010

30. Nikolay B, Brooker SJ, Pullan RL. Sensitivity of diagnostic tests for human soil-transmitted helminth infections: a meta-analysis in the absence of a true gold standard. Int J Parasitol. 2014;44:765-774. doi:10.1016/j.ijpara.2014.05.009

31. Beg M, Zeeshan M, Zafar A, et al. Use of Parasep filter fecal concentrator tubes" for the detection of intestinal parasites in stool samples under routine conditions. Indian $J$ Pathol Microbiol. 2011;54:121. doi:10.4103/0377-4929.77358

32. Khanna V, Sagar S, Khanna R, Chawla K. A comparative study of formalin-ethyl acetate sedimentation technique and Mini Parasep ${ }^{\circledR}$ solvent-free method in the rapid diagnosis of intestinal parasites. Trop Parasitol. 2018;8:29-32. doi:10.4103/tp.TP_44_17

33. Mewara A, Khurana S, Gupta S, Munda VS, Singh S, Sehgal R. Diagnostic Performance of Mini Parasep ${ }^{\circledR}$ Solvent-Free Foecal Parasite Concentrator for the Diagnosis of Intestinal Parasitic Infections. Indian J Med Microbiol. 2019;37:381-386. doi:10.4103/ijmm.IJMM_19_44
34. Useh M, Asuquo A, Otu-Bassey I, Ubi O. Evaluation of the Efficacy of the Mini Parasep SF Faecal Parasite Concentrator; A New Technique against the Direct Smear and Formol Ether Concentration Technique for the Detection of Intestinal Parasites in Stool. J Med Lab Sci. 2011;20:458.

35. Levecke B, Montresor A, Albonico M, et al. Assessment of Anthelmintic Efficacy of Mebendazole in School Children in Six Countries Where Soil-Transmitted Helminths Are Endemic. PLoS Negl Trop Dis. 2014;8(10):e3204. doi:10.1371/journal.pntd.0003204

36. David ED, Lindquist WD. Determination of the specific gravity of certain helminth eggs using sucrose density gradient centrifugation. J Parasitol. 1982;68:916-919. doi:10.1186/1756-3305-5-288

37. Levecke B, Cools P, Albonico M, et al. Identifying thresholds for classifying moderate-to-heavy soil-transmitted helminth intensity infections for FECPAKG2, McMaster, Mini-FLOTAC and qPCR. PLoS Negl Trop Dis. 2020;14(7):e0008296. doi:10.1371/journal.pntd.0008296

38. Knopp S, Glinz D, Rinaldi L, et al. FLOTAC: a promising technique for detecting helminth eggs in human faeces. Trans $R$ Soc Trop Med Hyg. 2009;103:1190-1194. doi:10.1016/j.trstmh.2009.05.012

39. Lim MD, Brooker SJ, Belizario VY, et al. Diagnostic tools for soil-transmitted helminths control and elimination programs: a pathway for diagnostic product development. PLoS Negl Trop Dis. 2018;12(3):e0006213. doi:10.1371/journal.pntd.0006213

40. Techion: FECPAKG2: better testing for better performance. Available from: https://www.techion.com/FECPAKG2. Accessed December 17, 2020.

41. Ayana M, Vlaminck J, Cools P, et al. Modification and optimization of the FECPAKG2 protocol for the detection and quantification of soil-transmitted helminth eggs in human stool. PLoS Negl Trop Dis. 2018;12(10):e0006655. doi:10.1371/journal.pntd.0006655

42. Moser W, Bärenbold O, Mirams GJ, et al. Diagnostic comparison between FECPAKG2and the Kato-Katz method for analyzing soil-transmitted helminth eggs in stool. PLoS Negl Trop Dis. 2018;12(6):e0006562. doi:10.1371/journal.pntd.0006562

43. Sukas S, van Dorst B, Kryj A, Lagatie O, De Malsche W, Stuyver LJ. Development of a lab-on-a-disk platform with digital imaging for identification and counting of parasite eggs in human and animal stool. Micromachines. 2019;10:852. doi:10.3390/mi10120852

44. Requena-Méndez A, Chiodini P, Bisoffi Z, Buonfrate D, Gotuzzo E, Muñoz J. The Laboratory Diagnosis and Follow Up of Strongyloidiasis: a Systematic Review. PLoS Negl Trop Dis. 2013;7 (1):e2002. doi:10.1371/journal.pntd.0002002

45. Garcia LS. Diagnostic Medical Parasitology. Manual of Commercial Methods in Clinical Microbiology. In: Allan L, editor. Diagnostic Medical Parasitology. Washington, DC, USA: ASM Press; 2014:274-305. doi:10.1128/9781555817961.ch11

46. Hailegebriel T, Petros B, Endeshaw T. Evaluation of Parasitological Methods for the Detection of Strongyloides Stercoralis among Individuals in Selected Health Institutions In Addis Ababa, Ethiopia. Ethiop J Health Sci. 2017;27:515-522. doi:10.4314/ejhs.v27i5.10

47. Sato Y, Toma H, Kiyuna S, Shiroma Y. Gelatin particle indirect agglutination test for mass examination for strongyloidiasis. Trans $R$ Soc Trop Med Hyg. 1991;85:515-518. doi:10.1016/0035-9203(91)90240-Y

48. Silva LP, Da Costa Barcelos IS, Passos-Lima AB, Espindola FS, Barbosa Campos DM, Costa-Cruz JM. Western Blotting Using Strongyloides ratti Antigen for the Detection of IgG Antibodies as Confirmatory Test in Human Strongyloidiasis. Mem Inst Oswaldo Cruz. 2003;98:687-691. doi:10.1590/S0074-02762003000500017

49. Schaffel R, Nucci M, Carvalho E, Braga M, Portugal R, Pulcheri W. The value of an immunoenzymatic test (enzyme-linked immunosorbent assay) for the diagnosis of strongyloidiasis in patients immunosuppressed by hematologic malignancies. Am J Trop Med Hyg. 2001;65:346-350. doi:10.4269/ajtmh.2001.65.346

50. Arifin N, Hanafiah KM, Ahmad H, Noordin R. Serodiagnosis and early detection of Strongyloides stercoralis infection. J Microbiol Immunol Infect. 2019;52:371-378. doi:10.1016/j.jmii.2018.10.001 
51. Ravi V, Ramachandran S, Thompson RW, Andersen JF, Neva FA. Characterization of a recombinant immunodiagnostic antigen (NIE) from Strongyloides stercoralis L3-stage larvae. Mol Biochem Parasitol. 2002;125:73-81. doi:10.1016/S0166-6851(02)00214-1

52. Ramanathan R, Burbelo PD, Groot S, Iadarola MJ, Neva FA, Nutman TB. A luciferase immunoprecipitation systems assay enhances the sensitivity and specificity of diagnosis of Strongyloides stercoralis infection. J Infect Dis. 2008;198:444-451. doi: $10.1086 / 589718$

53. Rascoe LN, Price C, Shin SH, Mcauliffe I, Priest JW, Handali S. Development of Ss-NIE-1 Recombinant Antigen Based Assays for Immunodiagnosis of Strongyloidiasis. PLoS Negl Trop Dis. 2015;9 (4):e0003694. doi:10.1371/journal.pntd.0003694

54. Yunus MH, Arifin N, Balachandra D, Anuar NS, Noordin R. Lateral Flow Dipstick Test for Serodiagnosis of Strongyloidiasis. Am J Trop Med Hyg. 2019;101:432-435. doi:10.4269/ajtmh.19-0053

55. Sykes AM, McCarthy JS. A coproantigen diagnostic test for Strongyloides infection. PLoS Negl Trop Dis. 2011;5(2):e955. doi:10.1371/journal.pntd.0000955

56. Lagatie O, Verheyen A, Van Hoof K, et al. Detection of Ascaris lumbricoides infection by ABA-1 coproantigen ELISA. PLoS Negl Trop Dis. 2020;14:e0008807. doi:10.1371/journal.pntd.0008807

57. Elsemore DA. Flynn LA Inventor; Idexx Laboratories Inc assignee. Device, kit and method for hookworm antigen capture and detection. 2007. United States patent US20080311557A1.

58. Lagatie O, Verheyen A, Van Asten S, et al. 2-Methyl-pentanoylcarnitine (2-MPC): a urine biomarker for patent Ascaris lumbricoides infection. Sci Rep. 2020;10:15780. doi:10.1038/s41598-020-72804-y

59. Lagatie O, Njumbe Ediage E, Pikkemaat JA, Djuardi Y, Stuyver LJ. 2-methyl butyramide, a previously identified urine biomarker for Ascaris lumbricoides, is not present in infected Indonesian individuals. Parasit Vectors. 2017;10(1):1-3. doi:10.1186/s13071017-2600-z

60. Ayana M, Cools P, Mekonnen Z, et al. Comparison of four DNA extraction and three preservation protocols for the molecular detection and quantification of soil- transmitted helminths in stool. PLoS Negl Trop Dis. 2019;13(10):e0007778. doi:10.1371/journal. pntd.0007778

61. O'Connell EM, Nutman TB. Review article: molecular diagnostics for soil-transmitted helminths. Am J Trop Med Hyg. 2016;95:508-514. doi:10.4269/ajtmh.16-0266

62. Grant JR, Pilotte N, Williams SA. A Case for Using Genomics and a Bioinformatics Pipeline to Develop Sensitive and Species-Specific PCR-Based Diagnostics for Soil-Transmitted Helminths. Front Genet. 2019;10:883. doi:10.3389/fgene.2019.00883

63. Howe KL, Bolt BJ, Cain S, et al. WormBase 2016: expanding to enable helminth genomic research. Nucleic Acids Res. 2016;44: D774-80. doi:10.1093/nar/gkv1217

64. Easton AV, Oliveira RG, O'Connell EM, et al. Multi-parallel qPCR provides increased sensitivity and diagnostic breadth for gastrointestinal parasites of humans: field-based inferences on the impact of mass deworming. Parasit Vectors. 2016;9:38. doi:10.1186/s13071-016-1314-y

65. Pilotte N, Papaiakovou M, Grant JR, et al. Improved PCR-based detection of soil transmitted helminth infections using a next-generation sequencing approach to assay design. PLoS Negl Trop Dis. 2016;10:e0004578. doi:10.1371/journal.pntd.0004578

66. Lynn M, Polderman Anton M, Vinkeles Melchers NVS. Diagnosing Polyparasitism in a High-Prevalence Setting in Beira, Mozambique: detection of Intestinal Parasites in Fecal Samples by Microscopy and Real-Time PCR. PLoS Negl Trop Dis. 2017;11(1):e0005310. doi:10.1371/journal.pntd.0005310

67. Verweij JJ, Canales M, Polman K, et al. Molecular diagnosis of Strongyloides stercoralis in faecal sam-ples using real-time PCR Trans R Soc Trop Med Hyg. 2009;103:342-346. doi:10.1016/j. trstmh.2008.12.001
68. Verweij JJ, Brienen EA, Ziem J, Yelifari L, Polderman AM, Van Lieshout L. Simultaneous detection and quantification of Ancylostoma duodenale, Necator americanus, and Oesophagostomum bifurcum in fecal samples using multiplex real-time PCR Am. J Trop Med Hyg. 2007;77:685-690. doi:10.4269/ajtmh.2007.77.685

69. Papaiakovou M, Gasser RB, Littlewood DTJ. Quantitative PCR-Based Diagnosis of Soil-Transmitted Helminth Infections: faecal or Fickle? Trends Parasitol. 2019;35(7):491-500. doi:10.1016/j. pt.2019.04.006

70. Dunn JC, Papaiakovou M, Han KT, et al. The increased sensitivity of qPCR in comparison to Kato-Katz is required for the accurate assessment of the prevalence of soil-transmitted helminth infection in settings that have received multiple rounds of mass drug administration. Parasit Vectors. 2020;13(1):324.

71. Papaiakovou M, Wright J, Pilotte N, et al. Pooling as a strategy for the timely diagnosis of soil-transmitted helminths in stool: value and reproducibility. Parasit Vectors. 2019;12(1):443.

72. Deng MH, Zhong LY, Kamolnetr O, Limpanont Y, Lv ZY. Detection of helminths by loop-mediated isothermal amplification assay: a review of updated technology and future outlook. Infect Dis Poverty. 2019;8:1-22. doi:10.1186/s40249-019-0530-z

73. Rashwan N, Diawara A, Scott ME, Prichard RK. Isothermal diagnostic assays for the detection of soil-transmitted helminths based on the SmartAmp2 method. Parasit Vectors. 2017;10:496. doi:10.1186/ s13071-017-2420-1

74. Shiraho EA, Eric AL, Mwangi IN, et al. Development of a Loop Mediated Isothermal Amplification for Diagnosis of Ascaris lumbricoides in Fecal Samples. J Parasitol Res. 2016;2016. doi:10.1155/2016/7376207

75. Mugambi RM, Agola EL, Mwangi IN, Kinyua J, Shiraho EA, Mkoji GM. Development and evaluation of a Loop Mediated Isothermal Amplification (LAMP) technique for the detection of hookworm (Necator americanus) infection in fecal samples. Parasit Vectors. 2015;8:574. doi:10.1186/s13071-015-1183-9

76. Watts MR, James G, Sultana Y, et al. A loop-mediated isothermal amplification (LAMP) assay for Strongyloides stercoralis in stool that uses a visual detection method with SYTO-82 fluorescent dye. Am J Trop Med Hyg. 2014;90:306-311. doi:10.4269/ajtmh.13-0583

77. Wakid MH. Fecal occult blood test and gastrointestinal parasitic infection. J Parasitol Res. 2010;2010:434801. doi:10.1155/2010/ 434801

78. Amoah ID, Singh G, Stenstrom TA, Reddy P. Detection and quantification of soil-transmitted helminths in environmental samples: a review of current state-of-the-art and future perspectives. Acta Trop. 2017;169:187-201. doi:10.1016/J.ACTATROPICA.2017.02.014

79. Oge H, Oge S. Quantitative comparison of various methods for detecting eggs of Toxocara canis in samples of sand. Vet Parasitol. 2000;92:75-79. doi:10.1016/S0304-4017(00)00276-4

80. World HEalth Organization (WHO) Guidelines for the safe use of wastewater, excreta and greywater - Volume 4. Available from: https://www.who.int/water_sanitation_health/publications/gsuweg4/ en/. Accessed January 18, 2021.

81. Environmental Protection Agency U. Environmental Regulations and Technology, Control of Pathogens and Vector Attraction in Sewage Sludge. Available from: https://www.epa.gov/sites/production/files/ 2015-07/documents/epa-625-r-92-013.pdf. Accessed January 18, 2021.

82. Jiménez B, Maya $\mathrm{C}$, Velásquez $\mathrm{G}$, et al. Identification and quantification of pathogenic helminth eggs using a digital image system. Exp Parasitol. 2016;166:164-172. doi:10.1016/j.exppara.2016.04.016

83. Rothrock MJ, Hiett KL, Kiepper BH, Ingram K, Hinton A. Quantification of Zoonotic Bacterial Pathogens within Commercial Poultry Processing Water Samples Using Droplet Digital PCR. $A d v$ Microbiol. 2013;03:403-411. doi:10.4236/aim.2013.35055 


\section{Publish your work in this journal}

Research and Reports in Tropical Medicine is an international, peerreviewed, open access journal publishing original research, case reports, editorials, reviews and commentaries on all areas of tropical medicine, including: Diseases and medicine in tropical regions; Entomology; Epidemiology; Health economics issues; Infectious disease; Laboratory science and new technology in tropical medicine;
Parasitology; Public health medicine/health care policy in tropical regions; and Microbiology. The manuscript management system is completely online and includes a very quick and fair peer-review system. Visit http://www.dovepress.com/testimonials.php to read real quotes from published authors. 